\title{
Comparison of total and cytoplasmic mRNA reveals global regulation by nuclear retention and miRNAs
}

\author{
Beata Werne Solnestam ${ }^{1 \dagger}$, Henrik Stranneheim ${ }^{1 \dagger}$, Jimmie Hällman ${ }^{1}$, Max Käller $^{1}$, Emma Lundberg ${ }^{2}$, \\ Joakim Lundeberg ${ }^{1 *}$ and Pelin Akan ${ }^{1}$
}

\begin{abstract}
Background: The majority of published gene-expression studies have used RNA isolated from whole cells, overlooking the potential impact of including nuclear transcriptome in the analyses. In this study, mRNA fractions from the cytoplasm and from whole cells (total RNA) were prepared from three human cell lines and sequenced using massive parallel sequencing.

Results: For all three cell lines, of about 15000 detected genes approximately 400 to 1400 genes were detected in different amounts in the cytoplasmic and total RNA fractions. Transcripts detected at higher levels in the total RNA fraction had longer coding sequences and higher number of miRNA target sites. Transcripts detected at higher levels in the cytoplasmic fraction were shorter or contained shorter untranslated regions. Nuclear retention of transcripts and mRNA degradation via miRNA pathway might contribute to this differential detection of genes. The consequence of the differential detection was further investigated by comparison to proteomics data. Interestingly, the expression profiles of cytoplasmic and total RNA correlated equally well with protein abundance levels indicating regulation at a higher level.
\end{abstract}

Conclusions: We conclude that expression levels derived from the total RNA fraction be regarded as an appropriate estimate of the amount of mRNAs present in a given cell population, independent of the coding sequence length or UTRs.

Keywords: Differential detection, Gene expression, Nuclear retention, miRNA regulation, RNA-Seq

\section{Background}

The advent of sequence-based assays of transcriptomes (RNA-Seq) has allowed better quantification of mRNA, with less bias and greater dynamic range than microarrays [1,2]. However RNA-Seq is undergoing a rapid evolution, and the impact of basic experimental design on data quality is still under investigation.

The majority of published transcriptome data have used RNA extracted from the whole cell (total RNA), assuming a negligible contribution of nuclear RNA to the total RNA population. This assumption has been

\footnotetext{
* Correspondence: joakim.lundeberg@scilifelab.se

${ }^{\dagger}$ Equal contributors

'KTH Royal Institute of Technology, Science for Life Laboratory (SciLifeLab Stockholm), School of Biotechnology, Division of Gene Technology, SE-171 65, Solna, Sweden

Full list of author information is available at the end of the article
}

challenged by Trask et al. [3], who demonstrated that the nuclear contribution does impact the gene expression profile when examining steady-state messenger RNA (mRNA) by microarray analysis. RNA extracted from the cytoplasmic fraction, which does not contain a nuclear RNA contribution, could also be used for RNASeq experiments. However, none of the available studies on RNA-Seq quantification have compared the use of total RNA with the use of cytoplasmic RNA.

During RNA synthesis, mRNAs are transcribed, spliced, capped, and polyadenylated in the nucleus and the resulting steady-state RNA is transported from nucleus to cytoplasm via nuclear pore complexes for translation. Messenger ribonucleoproteins are co-transcriptionally recruited to mRNA, and direct the export of mRNAs via their interaction with mRNA export factors and nuclear pore complexes [4]. This

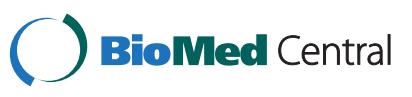


process is regulated at many levels, yielding a dynamic steady-state mRNA population that is maintained by synthesis and turnover, at varying rates for each individual transcript [5]. The rate of transportation from nucleus to cytoplasm can affect the amount of transcript detected in both the total and the cytoplasmic fractions, and hence might bias measurements of transcript levels. It has previously been shown that mRNA molecules that are not of immediate need to produce proteins are retained in the nucleus [6,7]. In addition to nuclear retention, the gene level is also regulated by other mechanisms and one of them is the degradation of mRNA by the exosome complex $[8,9]$.

It is known that the levels of mRNA and protein abundance in cells are modestly correlated [10-12]. One can argue that cytoplasmic RNA is a better proxy for protein levels since the cytoplasmic fraction contains only mature RNA; unlike total RNA, which also contains nuclear RNA. Validation of this argument will require studies that assess how well the levels of total RNA and cytoplasmic RNA are correlated with protein abundance.

To investigate the impact of nuclear transcripts present in total RNA, we compared the expression levels of genes obtained from the total fraction with those obtained from the cytoplasmic fraction. We performed poly(A)+ RNA-Seq experiments on three human cancer cell lines (A-431, U-2 OS, and U-251MG) on cytoplasmic and total RNA fractions in quadruplicates. We investigated the effect of the length and structure of untranslated regions and the length of the coding sequences on the transcript levels in total and cytoplasmic RNA. miRNA-mediated degradation of transcripts and its role in transcript regulation was also investigated, as well as the effect on the correlation with protein levels. We present here an extensive study of RNASeq that compares gene expression levels from poly (A) isolated total and cytoplasmic RNA as well as their relation to protein levels.

\section{Results}

RNA from three different human cell lines (A-431, U2 OS, and U-251MG) was extracted from whole cells (total RNA) and from the cytoplasm (cytoplasmic RNA), which does not include nuclear transcripts. Each extraction was replicated four times. To ensure that the cytoplasmic fraction was pure from nuclear contamination, all extractions were analyzed using capillary electrophoresis (Additional file 1: Figure S1). The nuclear and cytoplasmic preparations had, in addition to the ribosomal peaks, discriminatory signature profiles in which the nuclear fractions contained an additional peak, which were present only in the total RNA preparation [3,13]. The strongest signal, at roughly 4000 nucleotides $(\sim 52 \mathrm{~s})$, was used to determine nuclear RNA presence. All of the total RNA samples displayed the signature peak, whereas the cytoplasmic fractions did not (except for one cytoplasmic U-2 OS sample, which was removed from further processing). The samples were then sequenced using massively parallel sequencing, and RPKM (Reads Per Kilobase of exon model per Million mapped reads) values were calculated [14]. Expression values were in excellent agreement between total and cytoplasmic preparations for every cell line (Pearson correlation coefficient $>0.93$ (Figure 1A and Additional file 2: Figure S2) and the distribution of the RPKM values between the total and cytoplasmic fractions did not differ significantly (Figure 1B). In all cell lines, the gene coverage was slightly higher in cytoplasmic RNA than in total RNA.

The DESeq algorithm was used to find sets of genes detected at different levels in cytoplasmic and in total RNA [15], hereafter referred to as differentially detected (DD) genes. A number of DD genes were identified between the total and cytoplasmic fractions within each cell line (Figure 2A-C). In A-431 and U-251MG, 18\% and $15 \%$ of the genes were detected in different amounts between total and cytoplasmic RNA of the approximately 15000 detected genes; whereas in U-2 OS, only $6 \%$ of the genes were differentially detected $(p<0.001$, based on three replicates for each RNA fraction). There were approximately as many genes detected at higher levels in total RNA (1380, 405, and 1072 in A-431, U-2 OS, and U-251MG, respectively) and in cytoplasmic RNA (1334, 512, and 1203 genes in A-431, U-2 OS, and U-251MG, respectively).

\section{Length and structure of untranslated regions influence nucleus-to-cytoplasm transportation rate of transcripts} Messenger RNAs vary in sequence and length and this can affect their rate of transportation to the cytoplasm. To investigate this, genes that were detected differentially-in one, two, or all three cell lines-were selected and classified into two groups: genes that had a higher number of copies in the total RNA fraction and genes that had a lower number of copies in the total RNA fraction and plotted separately (Figure 2A and B). Differential detection of genes in total or cytoplasmic RNA fractions relies on that total RNA fraction would contain all mature polyadenylated transcripts whether they were in the cytoplasm or in the nucleus of the cell, whereas the cytoplasmic fractions only contain transcripts already transported to the cytoplasm.

To study whether the lengths of untranslated regions (UTRs) could affect the transportation rate of transcripts, we compared the UTR and coding sequence lengths of differentially detected genes with those of genes exhibiting no differential detection. We found that 


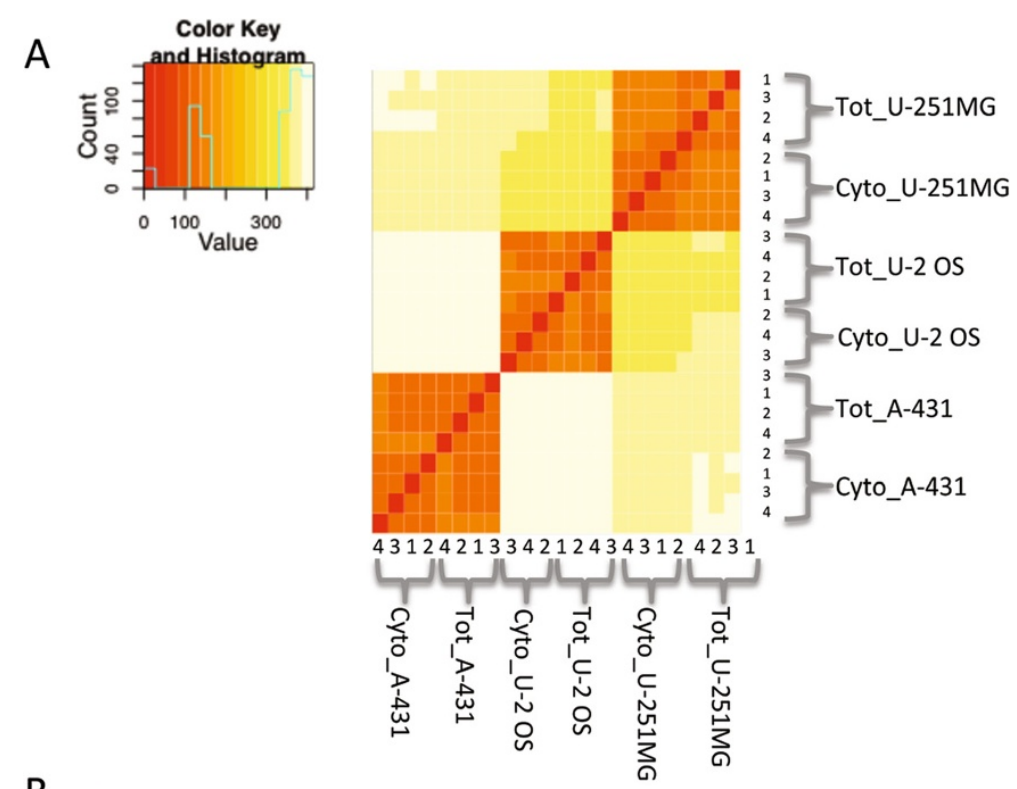

B

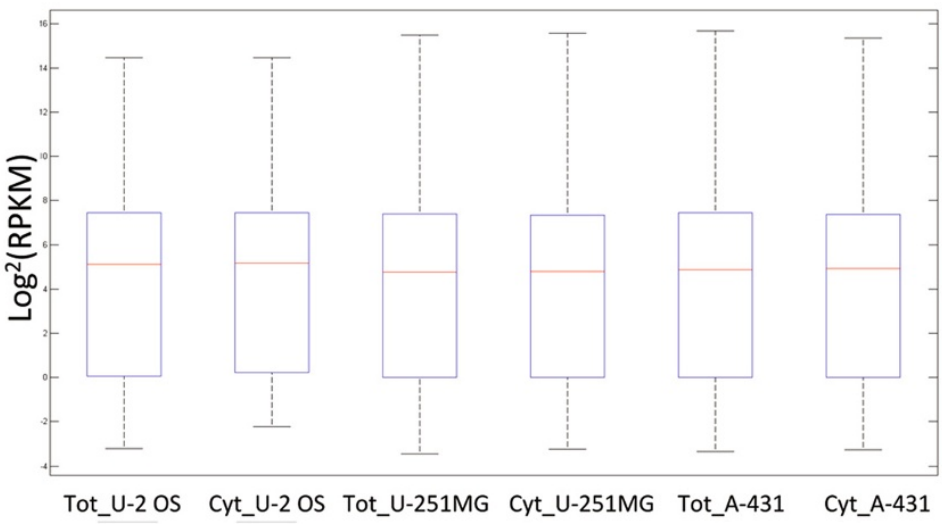

Figure 1 Distribution of gene expressions for the total and cytoplasmic preparation. A: Heatmap of sample preparation and cell lines. B The distribution of all RPKM values for total and cytoplasmic RNA for the three cell lines U-2 OS, U-251MG, and A-431. The mean RPKM values for total and cytoplasmic RNA of each cell line did not differ significantly $(p<0.05)$.

genes detected in higher amounts in total RNA $(p<$ 0.001 ) tended to have longer UTRs (Figure 3A and B) and that a significant proportion of longer transcripts were detected at lower levels in the cytoplasmic RNA (Figure $3 \mathrm{C}$ and Additional file 3 Figure S3C). This trend was consistent for genes that were differentially detected in one or more cell line (Figure 3 and Additional file 3: Figure S3A and B). We obtained UTR fold energies of 15,127 genes from the UCSC genome browser (calculated by Vienna RNA Package [16]). A more negative fold energy corresponds to a more structured sequence. Figure 3D-E and Additional file 4: Figure S4 show that genes that were detected at higher levels in total RNA had lower UTR fold energies (were more structured) than those with no differential detection. The data also show that the secondary structure of the 3' UTRs had a stronger effect compared to 5' UTRs.
Genes with higher numbers of miRNA target sites were detected in lower levels in the cytoplasmic RNA fraction compare to total RNA fraction

Transcripts that are degraded in the cytoplasm in high rates will also contribute to the differential detection since those degraded in cytoplasm will be detected at lower levels in the cytoplasmic RNA fraction compared to total RNA fraction. To investigate whether these genes have a higher number of microRNA (miRNA) targets, hence resulting in a higher probability for degradation when exported into the cytoplasm, an analysis comparing the number of miRNA targets per gene was performed. The same method to classify differentially detected genes (described in Figure 2) was used for the analysis. The miRNA data for the three cell lines (A-431, U-2 OS, U$251 \mathrm{MG}$ ) has been described elsewhere [17]. The list of 

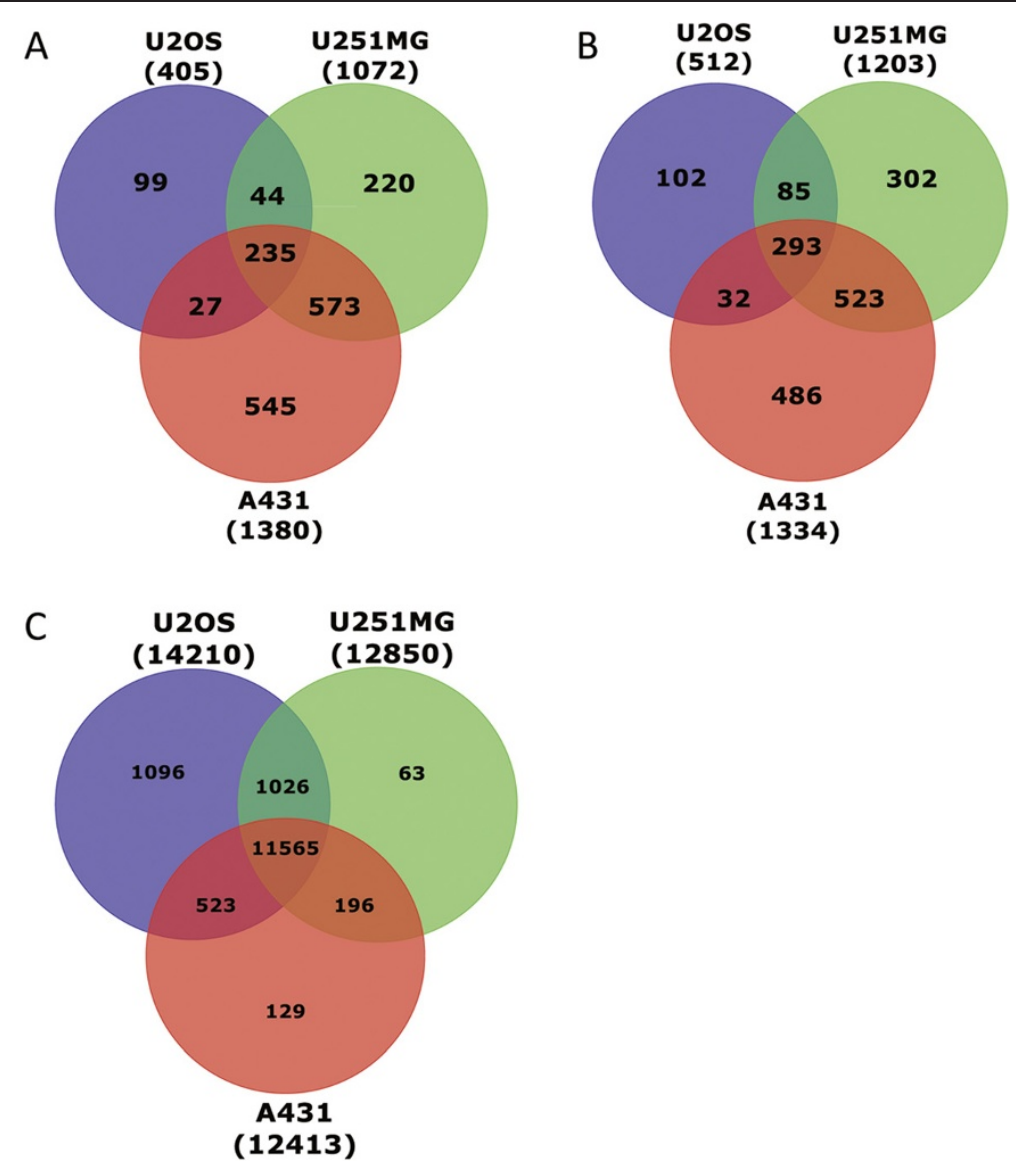

Figure 2 Number of differentially detected genes between the preparation methods for each cell line. A: Genes detected at a significantly higher level $(\mathbf{A})$, lower level $(\mathbf{B})$ or with no difference $(\mathbf{C})$ in total RNA compare to cytoplasmic RNA. The percentages of differentially detected genes were: A-431 18\%, U-251MG 15\%, and U-2 OS 6\%; calculated as the sum of genes at a higher and lower level divided by the total number of detected genes.

experimentally verified miRNAs per target gene was downloaded from miRTarBase [18] and compared with the list of expressed miRNA in each cell line. As described by Akan et al., the three cell lines have very similar miRNA profiles but U-2 OS have more uniquely expressed miRNAs. There was a significant difference ( $p<0,001)$ between the three groups of differentially detected genes for each cell line (Figure 4). The genes that had a higher number of transcripts in the total RNA fraction (Tot>Cyt) showed for all three cell lines a slightly higher number of miRNA targets than the genes that had a lower number of copies in the total RNA fraction (Tot<Cyt) and genes that where not differentially detected (No Diff). Interestingly, the cell line with a more pronounces difference between the groups, $\mathrm{U}-2 \mathrm{OS}$, is also the cell line that has more uniquely expressed miRNAs, and this could be the explanation for the slightly higher number of miRNA targets per gene seen in U-2 OS. Overall, the data suggests that the miRNA may be one of the contributing factors for differential detection of genes.
Correlation between mRNA and protein expression

A ratio-based correlation analysis (Spearman) was performed between protein abundance levels (detected by mass spectrometry for approximately 4700 proteins)[10] and the corresponding total and cytoplasmic mRNA levels, for each cell line. For U-2 OS, the correlation coefficient between protein abundance and total RNA was 0.6717 and between protein abundance and cytoplasmic RNA was 0.6790. The correlation coefficients for the other two cell lines (U-251MG and A-431) were very similar. There were no significant differences $(p=0.6)$ between total and cytoplasmic RNA levels in terms of correlation with protein abundance. The correlations were similar whether differentially detected genes were included or excluded, see Additional file 5: Table S1 for correlation coefficients between protein abundance and total and cytoplasmic RNA, respectively, for genes detected differentially in all three cell lines.

\section{Discussion}

When designing a gene expression experiment with the goal of measuring steady-state levels of mRNA, care 

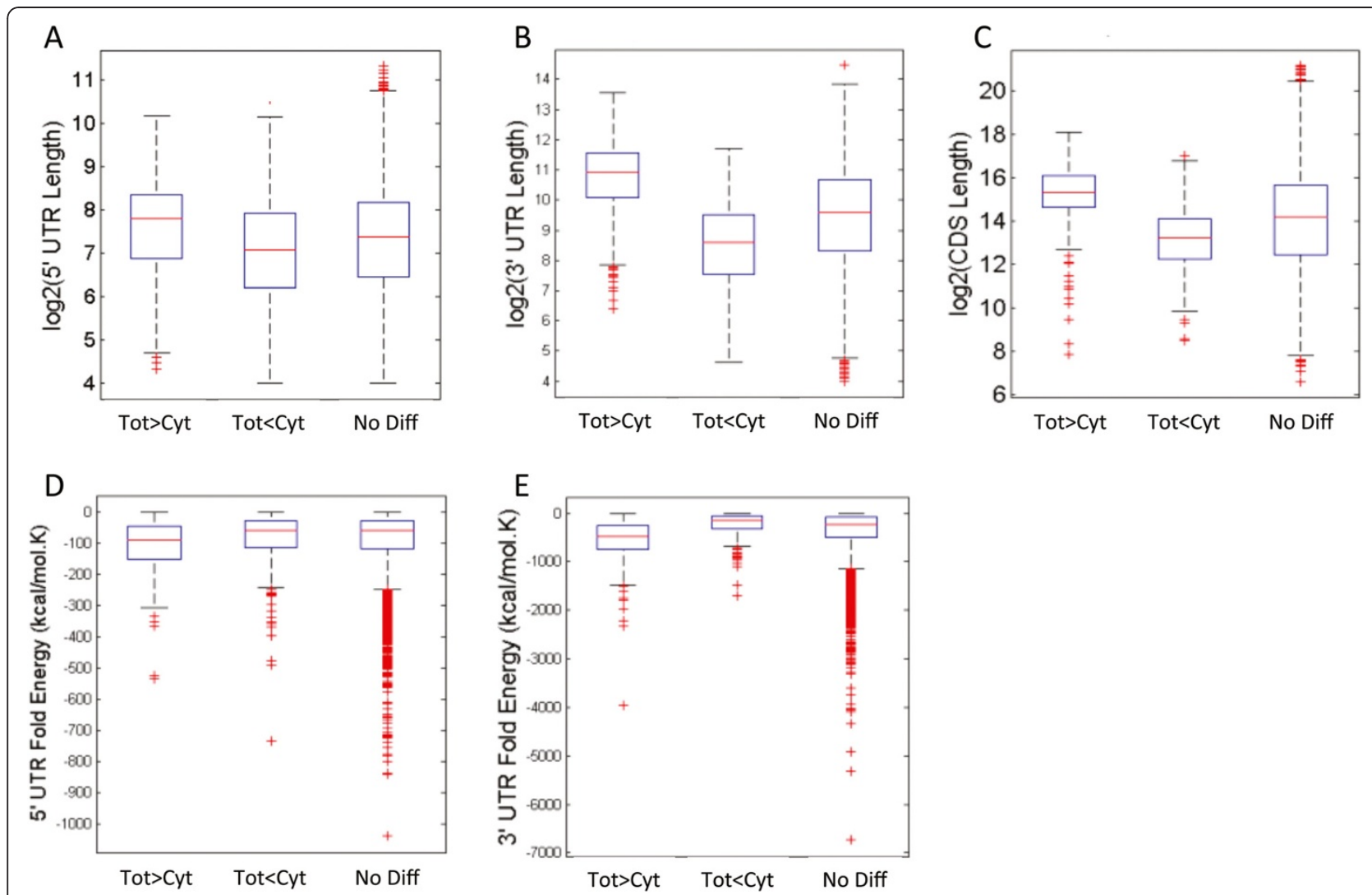

Figure 3 Boxplot showing length and fold energies of UTRs and coding sequence for all cell lines. Genes detected at a significantly higher level in total RNA than in cytoplasmic RNA (Tot>Cyt), lower level (Tot<Cyt), and genes with no significant differential detection (No Diff). A: Length of 5' UTRs. B: Length of 3' UTRs. C: Coding sequence length. D: Fold energy for 5' UTRs. E: Fold energy for 3' UTRs.

should be taken to isolate RNA from the correct cellular compartment. Currently, the majority of RNA-Seq experiments sequence mature transcripts (via poly-A tail enrichment) in the total RNA fraction, which also contain mature mRNA species to some degree [19]. Removing the $~ 5-10$ times more complex nuclear RNA [19] could reduce the overall complexity and enable deeper sampling of the remaining mRNA population and thus increase sensitivity. However, isolating the cytoplasmic RNA instead of total RNA is feasible when working with cell cultures, but for many other biological models are total RNA the only choice.

Despite the proposed advantage of sequencing only cytoplasmic RNA for cells in suspension, it is still not clear whether the cytoplasmic fraction represents the full complexity of the steady-state RNA of whole cells. One argument against using cytoplasmic RNA could be that the translation levels of certain transcripts might be regulated by their transportation rate from nucleus to cytoplasm [6,7]. Moreover, the transportation rate of transcripts from nucleus to cytoplasm could depend on particular properties of the transcript such as length or sequence.
Here, we investigated how the representations of transcripts differ between the cytoplasmic and total RNA fractions. There were 405, 1072, and 1380 transcripts in U-2 OS, U-251MG, and A-431 that were detected at higher levels in total RNA than in cytoplasmic RNA. This indicates that a significant proportion of the mature transcripts were retained in the nucleus, which then contributed to higher detection levels in the total RNA fraction since the cytoplasmic RNA lacked the mature transcripts from the nucleus. UTR fold energies can influence post-transcriptional regulation and it has been shown that UTR fold energies of mRNA transcripts are lower than those of random sequences of the same length with the same mononucleotide frequency $[20,21]$. Interestingly, most of the genes detected at higher level in total RNA had long and structured 5' and 3' UTR sequences as well as longer coding sequences, in all cell lines. Furthermore, it may cause an improper estimation of the RNA levels of these transcripts in the cytoplasmic fraction. Similarly, shorter genes or genes with shorter UTRs were overestimated in the cytoplasmic fraction. This mis-estimation could introduce biases and should be considered in the analysis of transcriptome. 


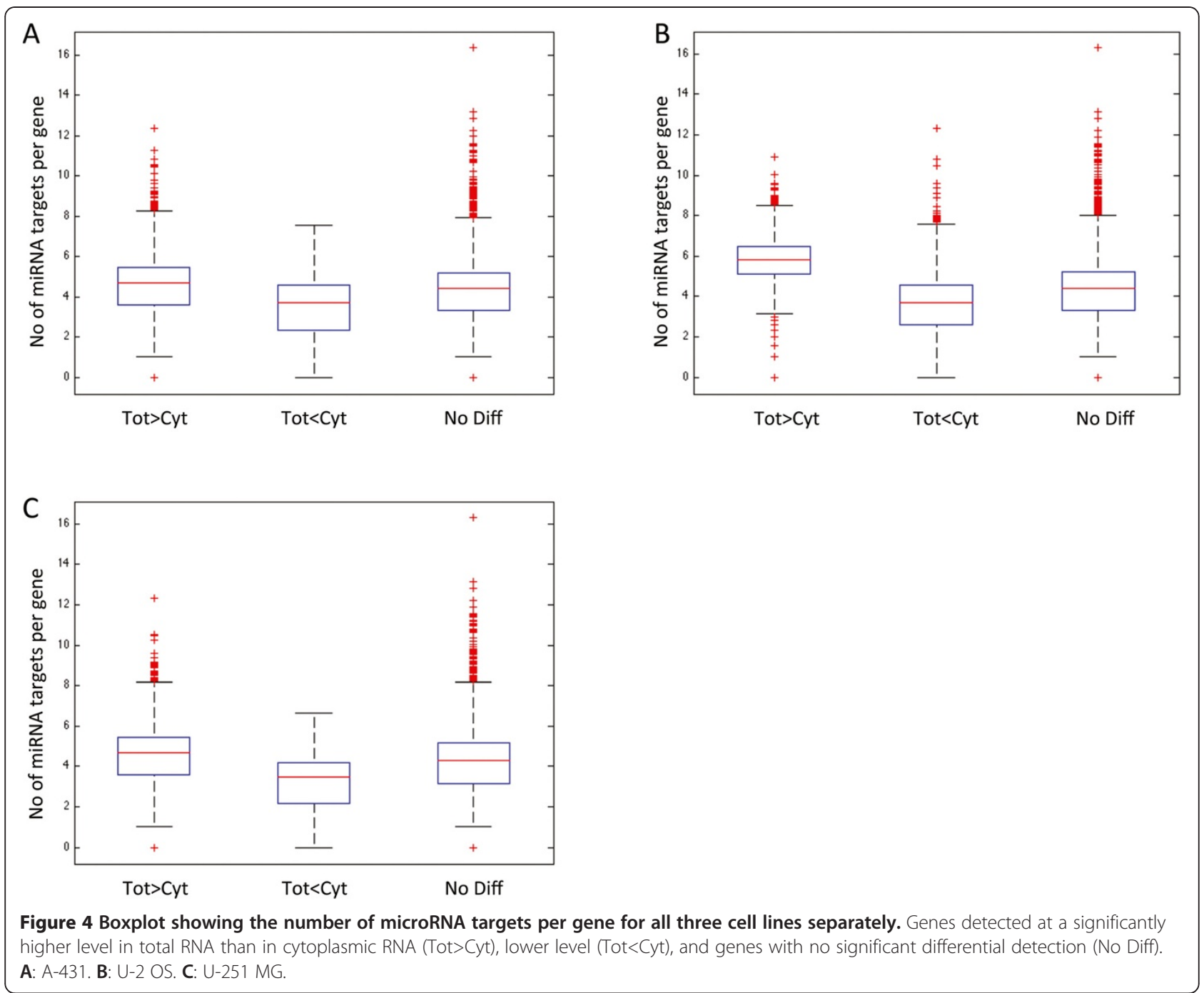

Hence, our data indicates that the transportation rate of transcripts from nucleus to cytoplasm depends on the sequence features of transcripts. Selective degradation of transcripts by for example the exosome complex and the half-life of transcripts cannot be ruled out as contributing factors. The results from the comparison of microRNA targets per gene for all three cell lines show that there is a higher number of microRNA targets per gene for genes detected differentially higher in the total RNA fraction compared to the cytoplasmic RNA fraction. This could indicate that these genes are subject to degradation to a higher degree when entering the cytoplasm. However this does not explain the higher number of genes with structured 5' UTR sequences as well as longer coding sequences in the total RNA fraction. Therefore, we propose that both nuclear retention and cytoplasmic RNA degradation via miRNAs are the main contributors to the differential detection of genes.
There were 512, 1203, and 1334 genes for U-2 OS, U-251MG, and A-431, respectively, that were detected at higher levels in cytoplasmic RNA than in total RNA. There is no obvious biological reason for this. However, a technical explanation can be suggested: owing to the lower representation of longer transcripts in the cytoplasmic fraction, there was relatively more sequencing space. This could have allowed for better coverage of shorter transcripts in cytoplasmic RNA than in total RNA. Indeed, most of the genes detected at higher levels in the cytoplasmic fraction had shorter coding lengths. However, not all the differentially detected genes were the same for all cell lines. This supports the fact that there are also cell-specific factors that affect the nuclear retention of transcripts, apart from transcript sequence and structure [7].

Our results have shown that the total and cytoplasmic fractions yield different representations of steady-state RNA levels. It can be argued that cytoplasmic 
polyadenylated RNA might correlate better with protein abundance levels if one assumes that the contribution of polyadenylated nuclear RNA to the steady-state mRNA levels in cytoplasm were not negligible. However, a previous study of mouse fibroblasts investigated mRNA and protein levels in relation to half-lives, transcription rates, and translational control and found that mRNA only explained around $40 \%$ of the variability in protein levels [22]. Our data show that cytoplasmic and total RNA correlated very similarly to protein abundance levels in all cell lines, and the correlation level is similar to what have previously been published [10]. This indicates that the neither nucleus-to-cytoplasm transportation rate nor the miRNA mediated degradation of transcripts affect protein abundance at a global level. However, future studies with synchronized cells and different time points would shed some more light upon the correlation between the RNA and protein population in a cell. Furthermore, including all transcripts and not only polyadenylated RNA would give amore complete overview of the RNA population in a given cell type.

\section{Conclusions}

Overall, our findings show that there are significant differences between total mRNA and cytoplasmic mRNA, which should be considered when comparing gene and protein expression patterns, and in general when using mRNA levels in different cellular compartments as a proxy for protein levels. Such efforts include whole genome/proteome comparisons, such as the human protein atlas initiative (www.proteinatlas.com) as well as other global efforts that correlate disease with genomic, transcriptomic, and proteomic information. Furthermore, our findings show that expression levels derived from the total RNA fraction be regarded as an appropriate estimate of the amount of mRNAs present in a given cell population, independent of the coding sequence length or UTRs.

\section{Methods}

\section{Cell cultivation}

The three human cell lines-the glioblastoma cell line U251MG (Prof. Bengt Westermark, Uppsala University), the epidermoid carcinoma cell line A-431 (DSMZ, Braunschweig, Germany), and the osteosarcoma cell line U-2 OS (ATCC-LGC, Middlesex, United Kingdom)were cultivated at $37^{\circ} \mathrm{C}$ in a $5 \% \mathrm{CO}_{2}$ environment in media suggested by the providers. Each cell-line was cultivated in four biological replicates and the cells were harvested during log-phase growth (60-70\% confluency).

\section{RNA isolation and purification}

RNA was extracted immediately after cell harvest. The total RNA fraction and the cytoplasmic fraction were extracted separately. The RNeasy Mini extraction kit (Qiagen, Hilden, Germany) was used according to the manufacturer's instructions for total RNA isolation. Cytoplasmic RNA was isolated according to the RNeasy protocol, except that the standard lysis buffer was exchanged for the lysis buffer RNL $[50 \mathrm{mM}$ Tris- $\mathrm{HCl}$, $\mathrm{pH}$ 8.0; $140 \mathrm{nM} \mathrm{NaCl} ; 1.5 \mathrm{mM} \mathrm{MgCl} 2 ; 0.5 \%$ (v/v) Nonidet P-40 $(1.06 \mathrm{~g} / \mathrm{ml})$; and $1 \mathrm{mM}$ DTT added just before use]. The extracted RNA samples were analyzed using an Experion automated electrophoresis system (Bio-Rad Laboratories, Hercules, CA, USA) with the standard-sensitivity RNA chip. All of the total RNA samples displayed a total RNA signature peak; as did one replicate of the cytoplasmic fraction of U-2 OS, which was discarded.

\section{Library preparation for sequencing}

Each cell-line was prepared in quadruplicate, with four biological replicates for total RNA and four for cytoplasmic RNA. A total of $3 \mu \mathrm{g}$ of high-quality RNA (RNA integrity number $=10$ ) per sample was used as input material for the mRNA sample preparations. The concentration and the RNA integrity number of the samples were determined from the run with the standardsensitivity RNA chip on the Experion automated electrophoresis system (Bio-Rad Laboratories, Hercules, CA, USA). The samples were bar-coded and prepared according to the protocol (Cat\# RS-930-1001) of the manufacturer (Illumina, San Diego, CA, USA) with the automated platform described previously [23].

\section{Clustering and sequencing}

The bar-coded libraries were clustered on a cBot clustergeneration system using an Illumina HiSeq single-read cluster-generation kit according to the manufacturer's instructions. The libraries were pooled together two and two in equal concentration for each lane on the flow cell, and sequenced as single reads to $100 \mathrm{bp}$ on an Illumina HiSeq 2000. All lanes were spiked with $1 \%$ phiX control library. The sequencing run was performed according to the manufacturer's instructions and generated a total of 908 million reads with a median of 40 million reads per sample and replicate that passed the Illumina Chastity filter; these reads were included in the study (Additional file 6: Table S2).

\section{Sequence analysis}

All sequences were aligned to the human genome reference hg19 with tophat [24,25] version 1.1 .4 and samtools [26] version 0.1.8 using tophat standard parameters except for: -solexa1.3-quals -p 8 -GTF Homo_sapiens. GRCh37.59.gtf. Annotations from ensembl and RefSeq, downloaded from UCSC Genome Browser, were used to assign features to genomic positions. Sequences aligned 
to the human genome were assigned to features and counted by HTSeq version 0.4 .6 with parameters: $-m$ intersection-strict -s no -t exon (Additional file 7: Table S3). The R/Bioconductor package DESeq [15] was used to call differential gene expression on counts generated by HTSeq. All biological replicates had $R^{2}$ (Spearman) correlation of gene expression (read counts) greater than 0.94 .

Reads per kilobase of exon per million mapped sequence reads (RPKM) values for features were calculated by rpkmforgenes.py using the parameters: -sam -gffann readcount. Estimations of intergenic expression levels for each replicate were calculated by rpkmforgenes.py and the $\mathrm{R}$ script cut_off.1.0.R (Additional file 8: Table S4) [27].

Reads were trimmed to determine the effect of sequencing length on the number of called differentially expressed genes using a custom perl script: trim_length. $\mathrm{pl}$, which is available on github (https://github.com/ henrikstranneheim).

Analysis of gene categories and pathways was performed by WebGestalt2 [28] with parameters: Id Type: ensembl_gene_stable_id, Ref Set: entrezgene, Significance Level: Top10, Statistics Test: Hypergeometric, MTC: BH, Minimum: 2.

5' and 3' UTR lengths and coding sequences were downloaded from UCSC. Lengths and fold energies were calculated with the Vienna RNA Package [16].

\section{Mass spectrometry data}

The protein data used in this study were generated in a previous study by Lundberg et al. [10], where a deep proteomic analysis was performed on the same three cell lines (A-431, U-2 OS, and U-251MG) used in this study. The cell lines were cultivated with amino acids with different isotopes and analyzed by mass spectrometry using a triple-SILAC method [29-31].

\section{Additional files}

Additional file 1: Figure S1. Overlays of electrophoresis diagrams of purified RNA. Total RNA and cytoplasmic RNA, showing the two ribosomal peaks (18S and 28S). In addition, the total RNA contains a nucleus-specific peak at roughly 4000 nucleotides ( 52 s), marked with an arrow in the upper diagram, which is missing in the cytoplasmic RNA fraction.

Additional file 2: Figure S2. Scatter plots of gene expression levels between total and cytoplasmic RNA. The median of the gene expression values of total and cytoplasmic RNA. The Pearson correlation coefficient $R^{2}$ is displayed in each scatter plot. A: U-2 OS, B: U-251 MG, C: A-431.

Additional file 3: Figure S3. Boxplot showing the length and coding sequence for all three cell lines. Genes detected at a significantly higher level in total RNA than in cytoplasmic RNA (Tot>Cyt), lower level (Tot<Cyt), and genes with no significant differential detection (No Diff). A: Length of 5' UTRs. B: Length of 3' UTRs. C: Coding sequence length.
Additional file 4: Figure S4. Boxplot showing the fold energies of UTRs for all three cell lines. Genes detected at a significantly higher level in total RNA than in cytoplasmic RNA (Tot>Cyt), lower level (Tot<Cyt), and genes with no significant differential detection (No Diff). A: Fold energy of 5' UTRs. B: Fold energy of 3' UTRs.

Additional file 5: Table S1. Spearman correlation for differentially and not differentially detected genes in all three cell lines.

Additional file 6: Table S2. Summary of information from the sequencing run.

Additional file 7: Table S3. Reads assigned to features using HTSeq.

Additional file $\mathbf{8}$ Table S4. Estimation of intergenic expressions levels within replicates based on RPKM.

\section{Abbreviations}

bp: base pair; CDS: Coding sequence; DD: Differentially detected; miRNA: micro-RNA; RPKM: Reads per kilobase of exon model per million mapped reads; UTRs: Untranslated regions.

\section{Competing interests}

The authors declare that they have no competing interests.

\section{Author's contributions}

BWS participated in the design of the study, purified the RNA, prepared the Illumina mRNA sequencing libraries, performed bioinformatics, and participated in drafting the manuscript. HS participated in the design of the study, performed bioinformatics and statistical analyses, and drafted the manuscript. . JH did the miRNA preparation. MK contributed with reagents, materials, and analysis tools. EL contributed with the cell lines and the growth of the cells. PA performed bioinformatics and statistical analyses and participated in drafting the manuscript. $J$ participated in the design of the study and participated in drafting the manuscript. All authors read and approved the final manuscript.

\section{Acknowledgements}

We would like to acknowledge Mikaela Wiking for growing and harvesting the cells; and Science for Life Laboratory (SciLifeLab Stockholm), SNISS, and Uppmax for providing massive parallel sequencing and computational infrastructure. Funding was obtained from the Swedish Scientific Council (VR) and European Commission grants 257743 and 222913.

\section{Author details}

TTH Royal Institute of Technology, Science for Life Laboratory (SciLifeLab Stockholm), School of Biotechnology, Division of Gene Technology, SE-171 65, Solna, Sweden. ${ }^{2}$ KTH Royal Institute of Technology, Science for Life Laboratory (SciLifeLab Stockholm), School of Biotechnology, Division of Proteomics, SE-171 65, Solna, Sweden.

Received: 11 July 2012 Accepted: 22 October 2012

Published: 30 October 2012

References

1. Shendure J: The beginning of the end for microarrays? Nat Methods 2008, 5(7):585-587.

2. Mortazavi A, Williams BA, McCue K, Schaeffer L, Wold B: Mapping and quantifying mammalian transcriptomes by RNA-Seq. Nat Methods 2008, 5(7):621-628.

3. Trask HW, Cowper-Sal-lari R, Sartor MA, Gui J, Heath CV, Renuka J, Higgins AJ, Andrews P, Korc M, Moore $\mathrm{H}$, et al: Microarray analysis of cytoplasmic versus whole cell RNA reveals a considerable number of missed and false positive mRNAs. RNA 2009, 15(10):1917-1928.

4. Hieronymus H, Silver PA: Genome-wide analysis of RNA-protein interactions illustrates specificity of the mRNA export machinery. Nat Genet 2003, 33(2):155-161.

5. Garcia-Martinez J, Aranda A, Perez-Ortin JE: Genomic run-on evaluates transcription rates for all yeast genes and identifies gene regulatory mechanisms. Mol Cell 2004, 15(2):303-313.

6. Prasanth KV, Prasanth SG, Xuan Z, Hearn S, Freier SM, Bennett CF, Zhang $M Q$, Spector DL: Regulating gene expression through RNA nuclear retention. Cell 2005, 123(2):249-263. 
7. Yasuda Y, Miyamoto Y, Yamashiro T, Asally M, Masui A, Wong C, Loveland $\mathrm{KL}$, Yoneda $\mathrm{Y}$ : Nuclear retention of importin alpha coordinates cell fate through changes in gene expression. EMBO J 2012, 31(1):83-94.

8. Chen CY, Gherzi R, Ong SE, Chan EL, Raijmakers R, Pruijn GJ, Stoecklin G, Moroni C, Mann M, Karin M: AU binding proteins recruit the exosome to degrade ARE-containing mRNAs. Cell 2001, 107(4):451-464.

9. Schaeffer D, Tsanova B, Barbas A, Reis FP, Dastidar EG, Sanchez-Rotunno M, Arraiano $C M$, van Hoof A: The exosome contains domains with specific endoribonuclease, exoribonuclease and cytoplasmic mRNA decay activities. Nat Struct Mol Biol 2009, 16(1):56-62.

10. Lundberg E, Fagerberg L, Klevebring D, Matic I, Geiger T, Cox J, Algenas C, Lundeberg J, Mann M, Uhlen M: Defining the transcriptome and proteome in three functionally different human cell lines. Mol Syst Biol 2010, 6:450.

11. de Sousa Abreu R, Penalva LO, Marcotte EM, Vogel C: Global signatures of protein and mRNA expression levels. Mol Biosyst 2009, 5(12):1512-1526.

12. Maier T, Guell M, Serrano L: Correlation of mRNA and protein in complex biological samples. FEBS Lett 2009, 583(24):3966-3973.

13. Wang Y, Zhu W, Levy DE: Nuclear and cytoplasmic mRNA quantification by SYBR green based real-time RT-PCR. Methods 2006, 39(4):356-362

14. Ramskold D, Wang ET, Burge CB, Sandberg R: An abundance of ubiquitously expressed genes revealed by tissue transcriptome sequence data. PLoS Comp Biol 2009, 5(12):e1000598.

15. Anders $S$, Huber W: Differential expression analysis for sequence count data. Genome Biol 2010, 11(10):R106.

16. Hofacker IL: Vienna RNA secondary structure server. Nucleic Acids Res 2003, 31(13):3429-3431.

17. Akan P, Costea PI, Alexeyenko A, Hedberg L, Werne Solnestam B, Lundin S, Hällman J, Lundberg E, Uhlén M, Lundeberg J: A Comprehensive Analysis of the Genome, Transcriptome and Proteome Landscapes of Three Human Tumor Cell Lines Reveals That Genomic Alterations Function Co-operatively in Tumorigenesis. 2012. Submitted.

18. Hsu SD, Lin FM, Wu WY, Liang C, Huang WC, Chan WL, Tsai WT, Chen GZ, Lee CJ, Chiu CM, et al: miRTarBase: a database curates experimentally validated microRNA-target interactions. Nucleic Acids Res 2011 39(Database issue):D163-169.

19. Sippel AE, Hynes N, Groner B, Schutz G: Frequency distribution of messenger sequences within polysomal mRNA and nuclear RNA from rat liver. Eur J Biochem 1977, 77(1):141-151.

20. Clote P, Ferre F, Kranakis E, Krizanc D: Structural RNA has lower folding energy than random RNA of the same dinucleotide frequency. RNA 2005, 11(5):578-591.

21. Ringner M, Krogh M: Folding free energies of 5'-UTRs impact posttranscriptional regulation on a genomic scale in yeast. PLoS Comp Biol 2005, 1(7):e72.

22. Schwanhausser B, Busse D, Li N, Dittmar G, Schuchhardt J, Wolf J, Chen W, Selbach M: Global quantification of mammalian gene expression control. Nature 2011, 473(7347):337-342.

23. Stranneheim H, Werne B, Sherwood E, Lundeberg J: Scalable transcriptome preparation for massive parallel sequencing. PLoS One 2011, 6(7):e21910.

24. Langmead B, Trapnell C, Pop M, Salzberg SL: Ultrafast and memoryefficient alignment of short DNA sequences to the human genome. Genome Biol 2009, 10(3):R25.

25. Trapnell C, Pachter L, Salzberg SL: TopHat: discovering splice junctions with RNA-Seq. Bioinformatics 2009, 25(9):1105-1111.

26. Li H, Handsaker B, Wysoker A, Fennell T, Ruan J, Homer N, Marth G, Abecasis G, Durbin R: The Sequence Alignment/Map format and SAMtools. Bioinformatics 2009, 25(16):2078-2079.

27. Ramskold D, Wang ET, Burge CB, Sandberg R: An abundance of ubiquitously expressed genes revealed by tissue transcriptome sequence data. PLoS Comput Biol 2009, 5(12):e1000598.

28. Zhang B, Kirov S, Snoddy J: WebGestalt: an integrated system fo exploring gene sets in various biological contexts. Nucleic Acids Res 2005 33(Web Server issue):W741-748.

29. Wisniewski JR, Zougman A, Mann M: Combination of FASP and StageTipbased fractionation allows in-depth analysis of the hippocampal membrane proteome. J Proteome Res 2009, 8(12):5674-5678.
30. Wisniewski JR, Zougman A, Nagaraj N, Mann M: Universal sample preparation method for proteome analysis. Nat Methods 2009, 6(5):359-362.

31. Shevchenko A, Tomas H, Havlis J, Olsen JV, Mann M: In-gel digestion for mass spectrometric characterization of proteins and proteomes. Nat Protoc 2006, 1(6):2856-2860.

doi:10.1186/1471-2164-13-574

Cite this article as: Solnestam et al: Comparison of total and cytoplasmic mRNA reveals global regulation by nuclear retention and miRNAs. BMC Genomics 2012 13:574.

\section{Submit your next manuscript to BioMed Central and take full advantage of:}

- Convenient online submission

- Thorough peer review

- No space constraints or color figure charges

- Immediate publication on acceptance

- Inclusion in PubMed, CAS, Scopus and Google Scholar

- Research which is freely available for redistribution

Submit your manuscript at www.biomedcentral.com/submit
C Biomed Central 